Article

\title{
Efficient Biotransformation of Polysialogangliosides for Preparation of GM1 by Cellulosimicrobium sp. 21
}

\author{
Yan Zheng ${ }^{1, \dagger}$, Li Ji ${ }^{2, \dagger}$, Jiayi Leng ${ }^{2}$, Ye Yuan ${ }^{2}$, Honglei Chen ${ }^{2}$, Dongxia Gou ${ }^{2}$, Yufei Gao ${ }^{1, *}$ \\ and Yifa Zhou ${ }^{2, *}$
}

1 China-Japan Union Hospital, Jilin University, Changchun 130033, Jilin, China;

E-Mail: zhy19780603@aliyun.com

2 School of Life Sciences, Northeast Normal University, Changchun 130024, Jilin, China;

E-Mails: jil132@nenu.edu.cn (L.J.); lengjy366@nenu.edu.cn (J.L.); yuany268@nenu.edu.cn (Y.Y.); chenh1150@nenu.edu.cn (H.C.); goudx479@nenu.edu.cn (D.G.)

$\dagger$ These authors contributed equally to this work.

* Authors to whom correspondence should be addressed; E-Mails: gaoyufei1975@sina.cn (Y.G.); zhouyf383@nenu.edu.cn (Y.Z.); Tel./Fax: +86-431-8509-8212 (Y.Z.).

External Editor: Derek J. McPhee

Received: 1 September 2014; in revised form: 28 September 2014 / Accepted: 29 September 2014 / Published: 8 October 2014

\begin{abstract}
A new ganglioside transformed strain isolated from soil was identified as Cellulosimicrobium sp. 21. It produced a sialidase which transformed polysialo-gangliosides GD1 and GT1 into a monosialoterahexosylganglioside, i.e., ganglioside GM1. The sialidase had both NeuAc- $\alpha-2,3$ - and NeuAc- $\alpha-2,8$-sialidase activity without producing asiolo-GM1. The optimum conditions were evaluated and it was found that the transformation was optimally performed at $30{ }^{\circ} \mathrm{C}$ and $\mathrm{pH}$ 7.0. The substrate should be added at the beginning of the reaction and the concentration of substrate was $3 \%(\mathrm{w} / \mathrm{v})$. Under these optimum conditions, Cellulosimicrobium sp. 21 converted GD1 and GT1 into GM1 in inorganic medium in a $5 \mathrm{~L}$ bioreactor with the recovery rate of $69.3 \%$. The product contained $50.3 \%$ GM1 and was purified on silica to give the product with $95 \%$ of GM1 with a recovery rate of $30.5 \%$. Therefore, Cellulosimicrobium sp. 21 has potential to be applied in the production of GM1 in the pharmaceutical industry.
\end{abstract}


Keywords: polysialoganglioside; Cellulosimicrobium sp. 21; biotransformation; sialidase; GM1

\section{Introduction}

The gangliosides are a group of complex glycosphingolipids containing sialic acid, existing most prominently in cerebral and nervous tissues. They contain a lipophilic ceramide residue and a carbohydrate chain of diverse length and complexity. The major gangliosides within the gangliotetraose family are classified into GM, GD, GT, and GQ, with one, two, three and four sialic acid groups per molecule, respectively [1].

Gangliosides play an important role in a variety of biological processes involved in the development and maintenance of the brain [2]. GM1 can penetrate the blood-brain barrier and has been developed as a drug applied clinically for neurological disorders such as Alzheimer's disease, Parkinson's disease, spinal cord injury, and stroke [3]. GM1 is usually prepared from animal brains by solvent extraction followed by chromatographic purification. Because of its low content in animal brains industrial preparation to isolate GM1 directly is difficult [4,5]. Scientists have made more efforts to convert GD1 and GT1 to GM1 by selectively removing one or two sialic acid residues in order to increase the amount of GM1 [6-8]. The methods usually used for this transformation are hydrolysis catalyzed by acids or enzymes. Chemical methods have less selectivity and are highly polluting to the environment, therefore, biotransformations may have more potential in these processes because of their mild conditions, low pollution and high selectivity. Although several sialidases (E.C. 3.2.1.18) which transform GD1 and GT1 to GM1 are available commercially, such as sialidase from Clostridium perfringens $(C$. welchii) Type VI and $\alpha-(2-3,6,8,9)$ sialidase from Arthrobacter ureafaciens, the high cost of their production limits their application in industry. Thus, the use of sialidase-producing microorganisms to convert GD1 and GT1 into GM1 has caught researchers' attention. Some strains such as Pseudomonas sp. strain YF-2 [9], Brevibacterium casei [10,11] and Oerskovia xanthineolytica YZ-2 [12] have been found to produce sialidases which convert GD1 and GT1 to GM1, but the transformation efficiency and conditions still hinder their application in industry. It is necessary to find more microbes for the efficient transformation of GD1 and GT1. In this study, we reported a new strain of Cellulosimicrobium isolated from soil, which could covert polysialogangliosides to GM1 with more efficiency. Further, the optimum conditions for the transformation were evaluated and the products were identified.

\section{Results and Discussion}

\subsection{Screening of Microorganisms for Biotransformation}

By screening microbes isolated from soil with monitoring by TLC and HPLC analysis, we found four stains that could convert polysialogangliosides into GM1 (Table 1). As shown, the bacterium numbered 21 (No. 21) showed higher catalytic activity in the transformation of GD1 and GT1 into GM1 than others. But there was little digestion of GM1 after incubation for three days, indicating that 
No. 21 could not hydrolyze GM1 to asialo-GM1. Strain No. 21 was identified by 16S rDNA gene sequence analysis by Shangon Biotech Co. Ltd. (Shanghai, China), as Cellulosimicrobium, and named Cellulosimicrobium sp. 21. The 16S rDNA (1407 bp) sequence analysis was uploaded in GenBank (accession number: KF133829).

Table 1. Screening results of microbiotransformation of polysialogangliosides.

\begin{tabular}{ccc}
\hline Strain & Content of GM1 (w/w, \%) & Conversion Time (h) \\
\hline No. 2 & 45.5 & 72 \\
No. 4 & 37.0 & 72 \\
No. 5 & 46.1 & 48 \\
No. 21 & 46.2 & 20 \\
\hline
\end{tabular}

Three kinds of microorganisms have been reported for GM1 biotransformation [9-12]. Two of them used a transformed organic medium [10,12], and one used an inorganic medium. Pseudomonas sp. strain YF-2 was the first microorganism which was reported for GM1 biotransformation by Kyushu University [9]. The substrate which was used contained GD1a, GD1b and GT1b. It took 3 days to finish the transformation in inorganic medium at $25{ }^{\circ} \mathrm{C}$. The rate of biotransformation was $80 \%-90 \%$. The other two were both reported by East China University of Science and Technology. Both of the substrates were crude gangliosides which contained GM1, GD1a, GD1b and GT1b. Brevibacterium casei finished the transformation in the medium with yeast powder at $30{ }^{\circ} \mathrm{C}$ after $24 \mathrm{~h}[10,11]$. Oerskovia xanthineolytica YZ-2 finished the transformation in the medium with yeast powder at $37^{\circ} \mathrm{C}$ after $18 \mathrm{~h}$. The content of GM1 was increased from $9 \%$ in crude gangliosides to $45 \%$ with a yield of $70 \%(\mathrm{w} / \mathrm{w})$ [12]. Comparing to those microbes reported in the literature, the No. 21 strain was more efficient. No. 21 almost completely transformed GD1 and GT1 to GM1 in inorganic medium after $20 \mathrm{~h}$. The content of GM1 was increased from $11.1 \%$ to $46.1 \%$ with a yield of $72.2 \%$. Nearly all the GD1 and GT1b were transformed to GM1 at the end of biotransformation (Figure 1). Next the optimal conditions for the biotransformation were studied to increase the transformation rate.

Figure 1. HPLC analysis of biotransformation product. Standards (a); gangliosides mixture (b); biotransformation product (c). Eluent: acetonitrile-phosphate buffer. Flow rate $1 \mathrm{~mL} / \mathrm{min}$ at $20^{\circ} \mathrm{C}$ and detection $215 \mathrm{~nm}$.

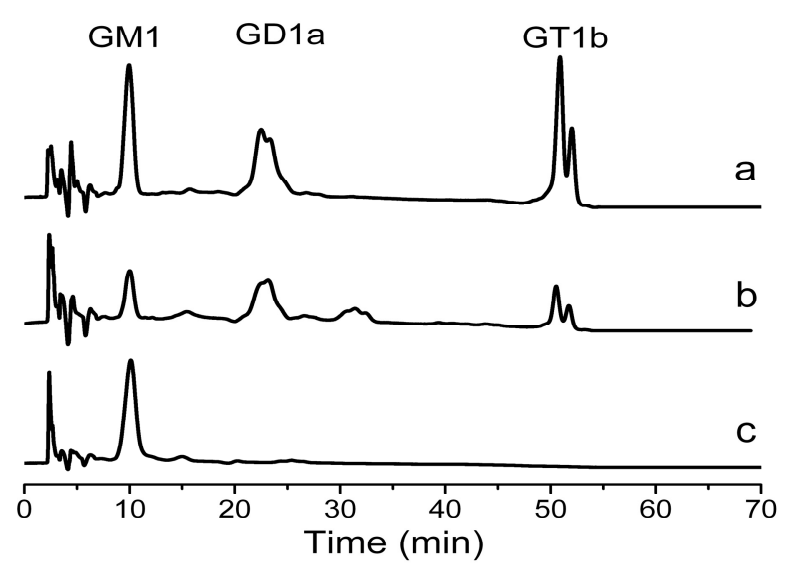




\subsection{Optimization of Conditions for Biotransformation}

In order to apply this biotransformation to the preparation of GM1, we evaluated the effects of the time of substrate addition, extra carbon supplements, temperature, $\mathrm{pH}$ and substrate concentration on biomass of microorganisms and the sialidase activity.

\subsubsection{Effect of Carbon Sources on Biotransformation}

In order to evaluate the effects of carbon sources on the growth of Cellulosimicrobium sp. 21 and its transformation activity, we cultured Cellulosimicrobium sp. 21 in medium containing polysialo-gangliosides as sole carbon source or the mixture of polysialogangliosides with glucose or glycerol $(1 \%, \mathrm{w} / \mathrm{v})$ as carbon source. As shown in Figure 2, as expected Cellulosimicrobium sp. 21 in control (None) grew only a little and produced no sialidase activity; it grew well and produced very high salidase transformation activity when polysialogangliosides were used as the sole carbon source (GLS); although glucose or glycerol increased the biomass, they decreased the sialidase activity (Gly and Glc). These results indicated that the extra carbon sources hindered salidase production. The sialidase was produced better from polysialogangliosides without other carbon sources, therefore, polysialo-gangliosides could be effectively transformed by Cellulosimicrobium sp. 21 when they were sole carbon source.

Figure 2. Effect of carbon source on transformation activity of Cellulosimicrobium sp. 21. Activity of sialidase (blank column), biomass (filled column). None, without carbon source; GLS, 1\% polysialogangliosides; Gly, 1\% polysialogangliosides and 1\% glycerol; Glc, $1 \%$ polysialogangliosides and $1 \%$ glucose. Biomass was determined by the absorption at $600 \mathrm{~nm}$. Activity of sialidase was determined by using 4-MU-NeuAc as fluorescent substrate and detection at $335 \mathrm{~nm} / 460 \mathrm{~nm}$.

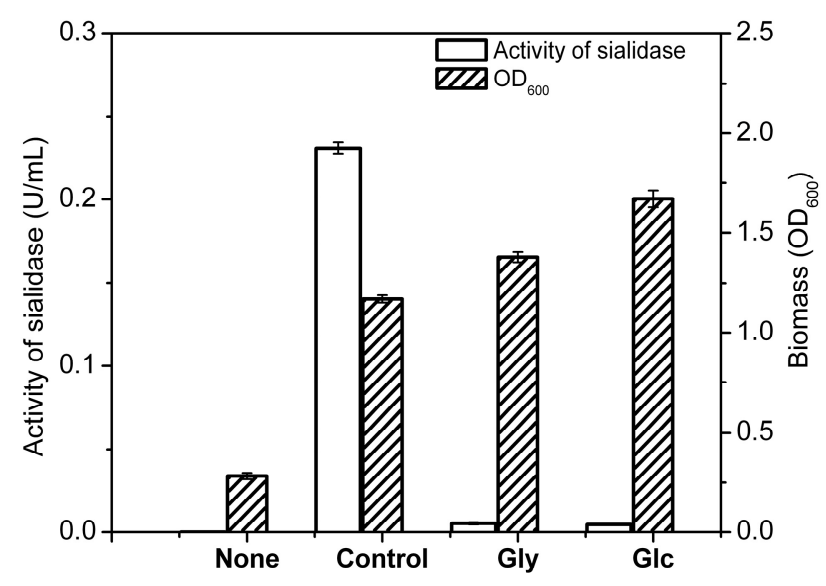

\subsubsection{The Optimal Time for Adding Substrate and Concentration of Substrate}

To test the optimal time for adding gangliosides, Cellulosimicrobium sp. 21 was cultured in medium with gangliosides as sole carbon source. The gangliosides were added to the medium at 0,2 , 4, 6, 8, 10 h. As shown in Figure 3, the activity of sialidase and the biomass of Cellulosimicrobium sp. 21 decreased significantly when ganglioside was added after $2 \mathrm{~h}$, indicating that the optimal time of adding the gangliosides mixture was at the beginning of the biotransformation. To evaluate the effect 
of the concentration of substrate, $0.5 \%, 1 \%, 3 \%, 5 \%, 7 \%$ and $9 \%$ of ganglioside in the medium were used to cultivate Cellulosimicrobium sp. 21 at $30{ }^{\circ} \mathrm{C}$ for $20 \mathrm{~h}$. As shown in Figure 4, the highest sialidase activity is observed in $3 \%$ of substrate.

Figure 3. Effect of substrate addition time on biotransformation. Activity of sialidase (blank column), biomass (filled column). Biomass was determined by the absorption at $600 \mathrm{~nm}$. Activity of sialidase was determined by using 4-MU-NeuAc as fluorescent substrate and detection at $335 \mathrm{~nm} / 460 \mathrm{~nm}$.

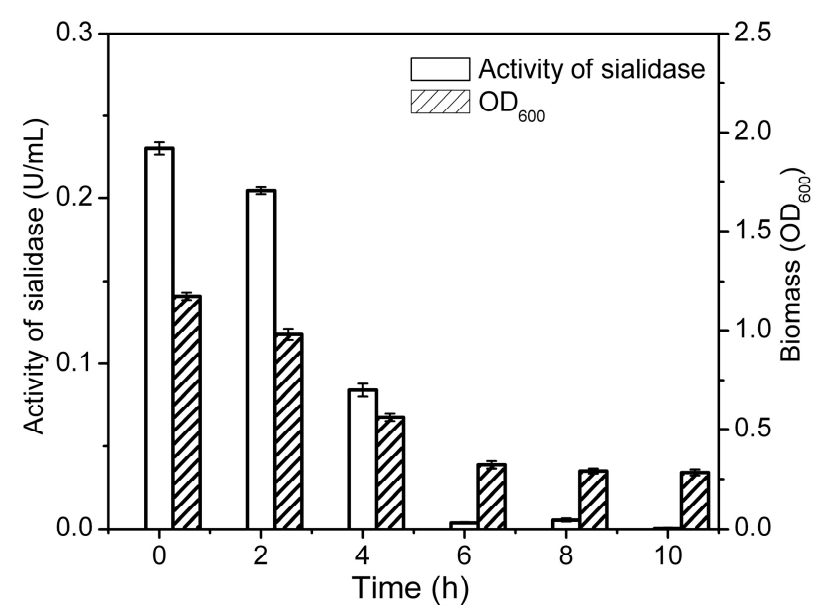

Figure 4. Effect of substrate concentration on biotransformation of Cellulosimicrobium sp. 21. Activity of sialidase (squares), Biomass (triangles). Biomass was determined by the absorption at $600 \mathrm{~nm}$. Activity of sialidase was determined by using 4-MU-NeuAc as fluorescent substrate and detection at $335 \mathrm{~nm} / 460 \mathrm{~nm}$.

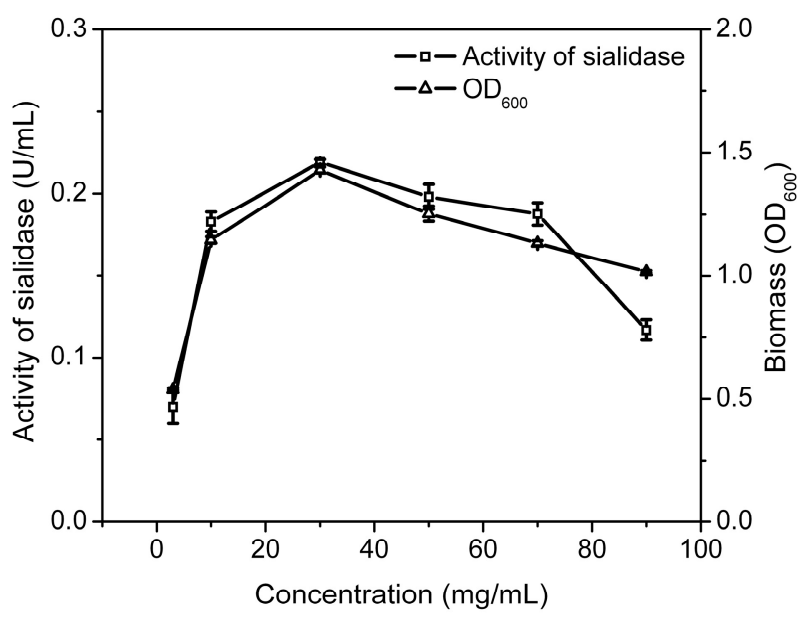

\subsubsection{The Optimal Biotransformation Temperature and $\mathrm{pH}$}

To evaluate the effects of temperature on the transformation, Cellulosimicrobium sp. 21 was cultured at different temperatures and then the biomass and the activity of sialidase were tested. As shown in Figure 5, both the biomass and the activity of sialidase achieved the highest values at $30{ }^{\circ} \mathrm{C}$, indicating that the optimal temperature for transformation was $30^{\circ} \mathrm{C}$. Cellulosimicrobium sp. 21 was incubated in different medium $\mathrm{pH}$ values (from 5 to 9 ) and then the growth and sialidase activity were 
determined. Figure 5 shows the effect of $\mathrm{pH}$ on the cell growth and the activity of sialidase. Both the biomass and the activity of sialidase achieved their highest values at $\mathrm{pH} 7$, indicating that $\mathrm{pH} 7$ was the optimal condition for the transformation.

Figure 5. Effect of temperature and $\mathrm{pH}$ on biotransformation activity of Cellulosimicrobium sp. 21. Activity of sialidase (squares), Biomass (triangles). Biomass was determined by the absorption at $600 \mathrm{~nm}$. Activity of sialidase was determined by using 4-MU-NeuAc as fluorescent substrate and detection at $335 \mathrm{~nm} / 460 \mathrm{~nm}$.
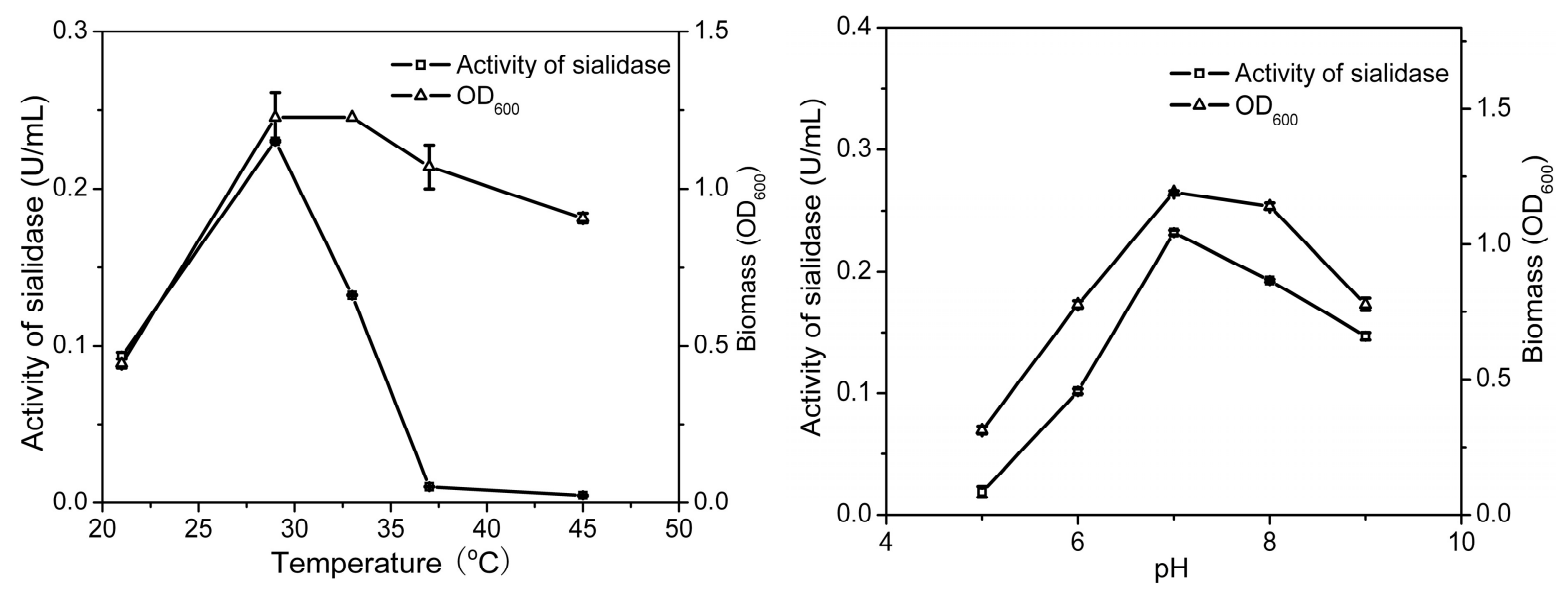

\subsection{Scale-Up of Conversion from Polysialogangliosides to GM1 in 5 L Bioreactor}

According to the optimal conditions evaluated in flask, the biotransformation of GD1 and GT1 to GM1 was performed in a $5 \mathrm{~L}$ bioreactor. After $20 \mathrm{~h}$ transformation, the product was centrifuged at $3200 \mathrm{~g}$ for $10 \mathrm{~min}$, dialyzed against distilled water and freeze dried, with a yield of $69.3 \%$. The content of GM1 in the product reached $50.3 \%$.

The biotransformation occurred in a simple inorganic medium with high efficiency; the purification of GM1 was relatively simple. GM1 was purified by silica chromatography only with a recovery rate of $30.5 \%$ and the purity was more than 95\% (Figures 6 and 7). The purification of GM1 from biotransformation systems in different ways has been reported. For Pseudomonas sp. strainYF-2, GM1 was purified from the culture supernatant by $\mathrm{C} 18$ reverse-phased chromatography, followed by DEAE-Sephadex A25 anion-exchange chromatography [9]. For Brevibacterium casei and Oerskovia xanthineolytica YZ-2, GM1 was purified from the culture supernatant by partitioning with a chloroform and methanol mixture, followed by acetone precipitation and silica gel chromatography $[10,12]$. The purification procedure was necessary to get rid of the residues of other gangliosides in the system, especially GD1a, GD1b and GT1b and the organic medium used in the biotransformation system. The biotransformation reported in this paper was very efficient and the products did not contain GD1a, GD1b and GT1b. In addition, the medium had a quite simple composition. Therefore, the purification of GM1 was relatively simple, requiring only silica gel chromatography. 
Figure 6. Result of the purification detected by TLC. Standard GM1 (a); ganglioside mixture (b); product after biotransformation (c); sample after purification (d). Solvent system: chloroform-methanol- $0.02 \%$ calcium chloride aqueous solution (60:36:8, v/v/v).

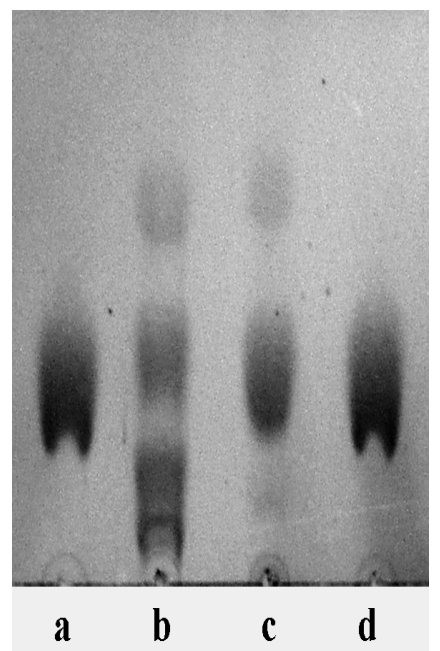

Figure 7. Result of GM1 purification. Standard of GM1 (a); sample after purification (b). Eluent: acetonitrile-phosphate buffer. Flow rate $1 \mathrm{~mL} / \mathrm{min}$ at $20^{\circ} \mathrm{C}$ and detection $215 \mathrm{~nm}$.

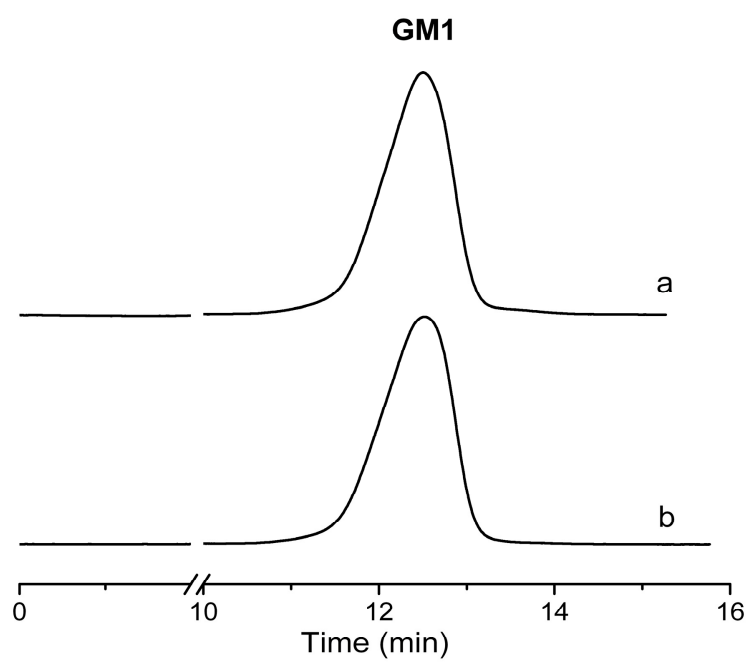

\subsection{Analysis of GM1}

The final product GM1 was identified by ESI-MS and ${ }^{13} \mathrm{C}-\mathrm{NMR}$. The characteristic negative ions $(\mathrm{M}-\mathrm{H})^{-}$were found at $\mathrm{m} / \mathrm{z} 1545.88$ and 1573.04 , which correspond to two molecular species of the monosialoganglioside GM1 with different ceramide (Figure 8). The ${ }^{13} \mathrm{C}-\mathrm{NMR}$ was recorded with purified GM1. The major chemical shifts are shown in Figure 9 and listed in Table 2. Compared with the results which have been reported in the literature, the final product was confirmed as GM1. 
Figure 8. The result of ESI-MS. The sample was dissolved in $\mathrm{MeOH}$ and detected in the negative-ion mode.

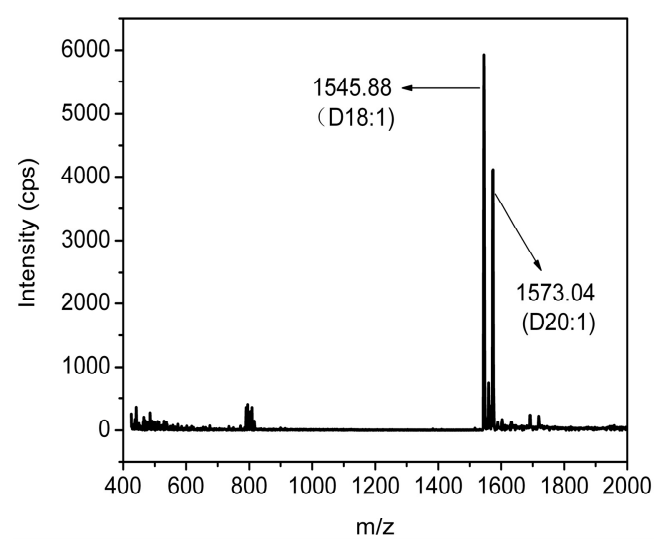

Figure 9. The result of ${ }^{13} \mathrm{C}-\mathrm{NMR}$. The sample was dissolved in $\mathrm{D}_{2} \mathrm{O}$ and detected at $150 \mathrm{MHz}$.

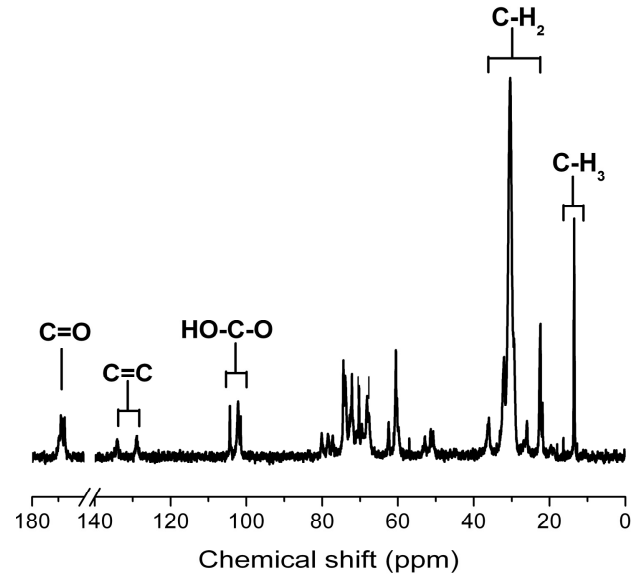

Table 2. ${ }^{13} \mathrm{C}-\mathrm{NMR}$ data for GM1 (150 MHz, solvent: $\left.\mathrm{D}_{2} \mathrm{O}\right)$.

\begin{tabular}{ccc}
\hline Residue & Carbon & Chemical Shift (ppm) \\
\hline$\beta$-D-Glc & $\mathrm{C}-1$ & 102.14 \\
& $\mathrm{C}-4$ & 78.49 \\
$\beta$-D-Gal & $\mathrm{C}-1$ & 102.17 \\
& $\mathrm{C}-3$ & 73.91 \\
& $\mathrm{C}-4$ & 77.19 \\
$\alpha$-D-NeuAc & $\mathrm{C}-1$ & 174.51 \\
& $\mathrm{C}-2$ & 101.49 \\
$\beta-\mathrm{D}-$ GalNAc & $\mathrm{N}-\mathrm{C}=\mathrm{O}$ & 174.23 \\
& $\mathrm{C}-1$ & 101.99 \\
$\beta$ & $\mathrm{C}-3$ & 80.09 \\
$\beta-\mathrm{D}-\mathrm{Gal}$ & $\mathrm{N}-\mathrm{C}=\mathrm{O}$ & 173.78 \\
Ceramide & $\mathrm{C}-1$ & 104.36 \\
& $\mathrm{C}-1-3$ & $51.31-68.16$ \\
& $\mathrm{C}-4$ & 134.06 \\
& $\mathrm{C}-5$ & 128.89 \\
& $\mathrm{C}-6-17$ & $21.83-30.40$ \\
Fatty acid chains & $\mathrm{C}-18$ & 13.60 \\
& $\mathrm{~N}-\mathrm{C}=\mathrm{O}$ & 173.78 \\
& $\mathrm{C}-2-15$ & $21.83-30.40$ \\
& $\mathrm{C}-16$ & 13.60 \\
\hline
\end{tabular}




\section{Experimental Section}

\subsection{Materials}

Ganglioside mixture containing GT1, GD1 and GM1 was extracted from pig brain as described in the literature [5]. Standards of GM1, GD1a, GT1b and 2'-(4-Methylumbelliferyl)- $\alpha$-D- $N$-acetyl-neuraminic acid sodium salt hydrate (4-MU-NeuAc) were purchased from Sigma-Aldrich (St. Louis, MO, USA). All other reagents and chemicals were commercially available reagents of analytical grade produced in China.

\subsection{Analytical Methods}

Thin layer chromatography (TLC) was carried out using a silica gel G 60 plate with the solvent system: chloroform-methanol- $0.02 \%$ calcium chloride aqueous solution $(60: 36: 8, \mathrm{v} / \mathrm{v} / \mathrm{v})$. The gangliosides developed on the plate were stained by spraying with resorcinol- $\mathrm{HCl}$ reagent, and then heating at $115{ }^{\circ} \mathrm{C}$ for $15 \mathrm{~min}$ [13]. The content of GM1 was determined by HPLC (Shimadzu, Kyoto, Japan) with an analytical Inertsil $\mathrm{NH}_{2}$ column $(4.6 \times 250 \mathrm{~mm}, 5 \mu \mathrm{m}$, Shimadzu, Kyoto, Japan $)$ as described by Gandini et al. [14]. ${ }^{13} \mathrm{C}-\mathrm{NMR}$ spectra were recorded on a Bruker AV 600 NMR spectrometer (Bruker, Benutzertagung Ettlingen, Germany) at $150 \mathrm{MHz}$ with $\mathrm{D}_{2} \mathrm{O}$ as the solvent. The electrospray ionization (ESI)-MS analysis were performed by the API $2000 \mathrm{LC} / \mathrm{MS} / \mathrm{MS}$ system (Applied Biosystems, Foster City, CA, USA) as described before [15,16].

\subsection{Strain Screening}

Soil samples collected from Changbai Mountain (Fusong, Jilin, China) were diluted with de-ionized water and centrifuged to remove particles. Then, the suspension were moved to a $1000 \mathrm{~mL}$ flask with $200 \mathrm{~mL}$ enrichment medium $\left(\mathrm{NaH}_{2} \mathrm{PO}_{4} \cdot 2 \mathrm{H}_{2} \mathrm{O}, 3.0 \mathrm{~g} / \mathrm{L}, \mathrm{Na}_{2} \mathrm{HPO}_{4} \cdot 12 \mathrm{H}_{2} \mathrm{O}, 10.9 \mathrm{~g} / \mathrm{L} ;\left(\mathrm{NH}_{4}\right)_{2} \mathrm{SO}_{4}, 10.0 \mathrm{~g} / \mathrm{L}\right.$; $\mathrm{pH} 7.0$ ) with $1 \%$ gangliosides mixture (w/v), and incubated at $30{ }^{\circ} \mathrm{C}, 200 \mathrm{rpm}$ for $24 \mathrm{~h}$ to enrich microorganisms. The broth of the microorganisms was checked by TLC at intervals.

The microorganisms screened above were streaked on LB agar plates. The isolated strains from LB agar plates were reexamined by incubating in enrichment medium with gangliosides mixture $(1 \% \mathrm{w} / \mathrm{v})$. GM1 contents after biotransformation was taken by HPLC. The strain having the highest activity of transformation was identified further by $16 \mathrm{~S}$ rDNA gene sequencing.

\subsection{S rDNA Gene Sequencing}

Chromosomal DNA of isolated strain for PCR was extracted by the method of Pospiech and Neumann [17]. 16S rDNA was cloned from chromosomal DNA using oligonucleotide primers: primer 1, 5'-AGAGTTTGATCCTGGCTCAG-3'; primer 2, 5'-GGTTACCTTGTTACGACTT-3'. The program for PCR was following: $94{ }^{\circ} \mathrm{C} 5 \mathrm{~min}, 29$ cycles of three steps $\left(94{ }^{\circ} \mathrm{C}\right.$ for $30 \mathrm{~s}, 54{ }^{\circ} \mathrm{C}$ for $30 \mathrm{~s}$ and $72{ }^{\circ} \mathrm{C}$ for $90 \mathrm{~s}$ ), and followed by $72{ }^{\circ} \mathrm{C}$ for $10 \mathrm{~min}$ additionally. The amplified DNA sequence was analyzed by Sangon Biotech Co. Ltd. (Shanghai, China). 


\subsection{Enzymatic Activity Assay of Sialidase}

Sialidase activity was determined by using 4-MU-NeuAc as fluorescent substrate. The reaction system was $200 \mu \mathrm{L}$ with $10 \mu \mathrm{L} 1 \mathrm{mmol} / \mathrm{L}$ 4-MU-NeuAc, $10 \mu \mathrm{L}$ sample solution, and $80 \mu \mathrm{L}$ of $200 \mathrm{mmol} / \mathrm{L}$ pH 5.5 sodium acetate buffer. The reaction system was used to measure fluorescence $(335 \mathrm{~nm} / 460 \mathrm{~nm})$ after incubation at $37{ }^{\circ} \mathrm{C}$ for $10 \mathrm{~min}$ and quenching with $1 \mathrm{~mol} / \mathrm{L}$-glycine-sodium hydroxide buffer solution (200 $\mu \mathrm{L}, \mathrm{pH} 10.4)$ addition. The control of the reaction system was boiled sample. One unit of the activity was defined as the amount of sialidase that hydrolyzed $1 \mu \mathrm{mol}$ substrate per minute [18].

\subsection{Cultivation and Biotransformation}

Cells were inoculated in a $25 \mathrm{~mL}$ flask with $10 \mathrm{~mL} \mathrm{LB}$ medium at $30{ }^{\circ} \mathrm{C}, 200 \mathrm{rpm}$ for $16 \mathrm{~h}$, and then transferred (5\% inoculation) into $10 \mathrm{~mL}$ enrichment medium containing $1 \%$ gangliosides mixture in a $25 \mathrm{~mL}$ flask for sialidase production at $30{ }^{\circ} \mathrm{C}, 200 \mathrm{rpm}$ for $20 \mathrm{~h}$. Both samples were collected for GM1 content and activity of sialidase determination during the biotransformation. The optimum conditions of biotransformation were tested, including the carbon source, the optimal time for adding substrate and concentration of substrate, the optimal temperature and $\mathrm{pH}$.

\subsection{Preparative Scale Biotransformation}

The biotransformation for preparation of GM1 was enlarged in a bioreactor (BIOTECH-5BG, Shanghai, China) equipped with DO and pH electrodes. Enrichment medium (2 L) was used for cell culture and GM1 conversion after sterilization at $115{ }^{\circ} \mathrm{C}$ for $20 \mathrm{~min}$. The Cellulosimicrobium sp. 21 was inoculated in $500 \mathrm{~mL}$ flask with $100 \mathrm{~mL} \mathrm{LB}$ medium at $30{ }^{\circ} \mathrm{C}, 200 \mathrm{rpm}$ for cultivating $16 \mathrm{~h}$, and then transferred (5\% inoculation) in bioreactor with $2 \mathrm{~L}$ enrichment medium containing $3 \%$ gangliosides mixture (containing $11 \%$ of GM1) extracted from pig brains. The medium was inorganic medium only with gangliosides. The conditions of transformation were at $30{ }^{\circ} \mathrm{C}, \mathrm{pH} 7.0,200 \mathrm{rpm}$ and $0.8 \mathrm{~L} / \mathrm{min}$ of air supplement. After $20 \mathrm{~h}$ transformation, the product was centrifuged at $3200 \times \mathrm{g}$ for $10 \mathrm{~min}$, dialyzed against distill water and freeze dried.

\subsection{Separation and Purification of GM1}

When the biotransformation was finished, cells were removed by centrifugation at $3200 \times g$ for 10 min. The supernatant was dialyzed against distilled water and freeze dried. The content of GM1 in the product was determined by HPLC. The purification of GM1 was performed by the method of Ledeen and $\mathrm{Yu}$ [19] with some modifications. The biotransformation product was dissolved in chloroform-methanol (4/1, v/v) and subjected to silica gel chromatography by first washing the column with chloroform-methanol $(4 / 1, \mathrm{v} / \mathrm{v})$ to remove impurities, then eluting with chloroform-methanol $(5 / 4, v / v)$ to give GM1.

\section{Conclusions}

A novel strain for GM1 production was isolated and named Cellulosimicrobium sp. 21. It can transfer polysialoganglioside to GM1 in simple inorganic medium and under mild conditions. The 
yield (69.3\%) was higher than those of biotransformations reported in the literature [9-12]. The biotransformation in simple inorganic medium resulted in a simpler product purification than those of similar biotransformation products in usual media. The purification was performed by chromatography on silica gel to get GM1 of over 95\% purity. Therefore, the method developed in this paper has potential for preparation of GM1 in industry.

\section{Acknowledgments}

This work was supported by the Chinese New Drug Creation and Manufacturing Program (2012ZX09502001-001), the Program for New Century Excellent Talents in University (NCET-11-0614), and Jilin Provincial Science \& Technology Department Program (201205005).

\section{Author Contributions}

Yan Zheng was responsible for data acquisition, analysis and manuscript preparation. Li Ji was responsible for the experimental design. Jiayi Leng and Ye Yuan were helped to analyze the data. Dongxia Gou and Honglei Chen worked on the preparation of the studied materials. Yifa Zhou and Yufei Gao were responsible for the programs and all experiments, critically revised the manuscript and provided final approval for the article.

\section{Conflicts of Interest}

The authors declare no conflict of interest.

\section{References}

1. McCluer, R.H. Chemistry of gangliosides. Chem. Phys. Lipids 1970, 5, 220-234.

2. Nekrasov, E.; Hubl, U. Gangliosides. In Sialobiology: Structure, Biosynthesis and Fuction; Tiralongo, J., Martinez-Duncker, I., Eds.; Bentham Science Publishers: Sharjah, UAE, 2013; Chapter 10, pp. 313-380.

3. Nobile-Orazio, E.; Carpo, M.; Scarlato, G. Gangliosides: Their role in clinical neurology. Drugs 1994, 47, 576-585.

4. Folch, J.; Lees, M.; Sloane-Stanley, G.H. A simple method for the isolation and purification of total lipids from animal tissues. J. Biol. Chem. 1957, 226, 497-509.

5. Svennerholm, L.; Fredman, P. A procedure for the quantitative isolation of brain gangliosides. Biochim. Biophys. Acta 1980, 617, 97-109.

6. Tettamanti, G.; Bonali, F.; Marchesini, S.; Zambotti, V. A new procedure for the extraction, purification and fractionation of brain gangliosides. Biochim. Biophys. Acta 1973, 296, 160-170.

7. Colarow, L.; Turini, M.; Teneberg, S.; Berger, A. Characterization and biological activity of gangliosides in buffalo milk. Biochim. Biophys. Acta 2003, 1631, 94-106.

8. Mauri, L.; Casellato, R.; Kirschner, G.; Sonnino, S. A procedure of the preparation of GM3 ganglioside from GM1-lactone. Glycoconj. J. 1999, 16, 197-203.

9. Fukano, Y.; Ito, M. Preparation of GM1 ganglioside with sialidase-producing marine bacteria as a microbial biocatalyst. Appl. Environ. Microbiol. 1997, 63, 1861-1865. 
10. Wang, X.D.; Yin, Z.; Peng, Y.F.; Shen, Y.L.; Wei, D.Z. Highly efficient conversion of polysialoganglioside to GM1 with Brevibacterium casei as a microbial biocatalyst. Biocatal. Biotransform. 2005, 23, 29-32.

11. Peng, Y.F.; Wang, X.D.; Wei, D.Z. Development of a large scale process for the conversion of polysialogangliosides to monosialotetrahexosylganglioside with a novel strain of Brevibacterium casei producing sialidase. Biotechnol. Lett. 2007, 29, 885-889.

12. Zhang, J.G.; Cao, D.; Shen, D.H.; Wang, X.D.; Wei, D.Z. Efficient conversion from polysialogangliosides to monosialotetrahexosylganglioside using Oerskovia xanthineolytica YZ-2. Bioprocess Biosyst. Eng. 2011, 34, 493-498.

13. Scandroglio, F.; Loberto, N.; Valsecchi, M.; Chigorno, V.; Prinetti, A.; Sonnino, S. Thin layer chromatography of gangliosides. Glycoconj. J. 2009, 26, 961-973.

14. Gangdini, C.; Kitsos, M.; Massolini, G.; Delorenzi, E.; Soldi, A.; Caccialanza, G.; Kirschner, G. Determination of gangliosides in parenteral dosage form by high-performance liquid chromatography. J. Pharm. Biomed. 1990, 8, 1063-1066.

15. Ikeda, K.; Shimizu, T.; Taguchi, R. Targeted analysis of ganglioside and sulfatide molecular species by LC/ESI-MS/MS with theoretically expanded multiple reaction monitoring. J. Lipid Res. 2008, 49, 2678-2689.

16. Hildebrandt, H.; Jonas, U.; Ohashi, M.; Klaiber, I.; Rahmann, H. Direct electrospary-ionization mass spectrometric analysis of the major ganglioside from crucian carp liver after thin layer chromatography. Comp. Biochem. Physiol. B 1999, 122, 83-88.

17. Pospiech, A.; Neumann, B. A versatile quick-prep of genomic DNA from gram-positive bacteria. Trends Genet. 1995, 11, 217-218.

18. Pukin, A.V.; Weijers, C.A.G.M.; Lagen, B.; Wechselberger, R.; Sun, B.; Gilbert, M.; Karwaski, M.F.; Florack, D.E.A.; Jacobs, B.C.; Tio-Gillen, A.P.; et al. GM3, GM2 and GM1 mimics designed for biosensing: Chemoenzymatic synthesis, target affinities and $900 \mathrm{MHz} \mathrm{NMR}$ analysis. Carbohydr. Res. 2008, 343, 636-650.

19. Ledeen, R.W.; Yu, R.K. Gangliosides: Structure, isolation, and analysis. Methods Enzymol. 1985, 83, 139-191.

Sample Availability: Sample of compound GM1 is available from the authors.

(C) 2014 by the authors; licensee MDPI, Basel, Switzerland. This article is an open access article distributed under the terms and conditions of the Creative Commons Attribution license (http://creativecommons.org/licenses/by/4.0/). 